\title{
Cochrane
}

Cochrane Database of Systematic Reviews

\section{Healthcare workers' perceptions and experience on using mHealth technologies to deliver primary healthcare services: qualitative evidence synthesis (Protocol)}

Odendaal WA, Goudge J, Griffiths F, Tomlinson M, Leon N, Daniels K

Odendaal WA, Goudge J, Griffiths F, Tomlinson M, Leon N, Daniels K.

Healthcare workers' perceptions and experience on using $\mathrm{mHealth}$ technologies to deliver primary healthcare services: qualitative evidence synthesis.

Cochrane Database of Systematic Reviews 2015, Issue 11. Art. No.: CD011942.

DOI: 10.1002/14651858.CD011942.

www.cochranelibrary.com

Healthcare workers' perceptions and experience on using mHealth technologies to deliver primary healthcare services: qualitative evidence synthesis (Protocol) 
TABLE OF CONTENTS

HEADER . . . . . . . . . . . . . . . . . . . . . . . . . . . . . . . . . . . . 1

ABSTRACT . . . . . . . . . . . . . . . . . . . . . . . . . . . . . . . . . . . . . . . . . .

BACKGROUND . . . . . . . . . . . . . . . . . . . . . . . . . . . . . . . . . . . . . .

OBJECTIVES . . . . . . . . . . . . . . . . . . . . . . . . . . . . . . . . . . . . . . .

METHODS . . . . . . . . . . . . . . . . . . . . . . . . . . . . . . . . . . . . . .

ACKNOWLEDGEMENTS . . . . . . . . . . . . . . . . . . . . . . . . . . . . . . . . . . . . . 6

REFERENCES . . . . . . . . . . . . . . . . . . . . . . . . . . . . . . . . . . . . . . 6

APPENDICES . . . . . . . . . . . . . . . . . . . . . . . . . . . . . . . . . . . . . 9

CONTRIBUTIONS OF AUTHORS . . . . . . . . . . . . . . . . . . . . . . . . . . . . . . . . . . . . 13

DECLARATIONS OF INTEREST . . . . . . . . . . . . . . . . . . . . . . . . . . . . . . . . . 13

SOURCES OF SUPPORT . . . . . . . . . . . . . . . . . . . . . . . . . . . . . . . . . . . . . . . . . .

Healthcare workers' perceptions and experience on using mHealth technologies to deliver primary healthcare services: qualitative evidence synthesis (Protocol)

Copyright $\odot 2015$ The Cochrane Collaboration. Published by John Wiley \& Sons, Ltd. 


\title{
Healthcare workers' perceptions and experience on using mHealth technologies to deliver primary healthcare services: qualitative evidence synthesis
}

\author{
Willem A Odendaal ${ }^{1}$, Jane Goudge ${ }^{2}$, Frances Griffiths ${ }^{3}$, Mark Tomlinson ${ }^{4}$, Natalie Leon ${ }^{1}$, Karen Daniels ${ }^{1}$ \\ ${ }^{1}$ Health Systems Research Unit, Medical Research Council of South Africa, Tygerberg, South Africa. ${ }^{2}$ Centre for Health Policy, School \\ of Public Health, University of the Witwatersrand, Johannesburg, South Africa. ${ }^{3}$ Warwick Medical School, University of Warwick, \\ Coventry, UK. ${ }^{4}$ Department of Psychology, University of Stellenbosch, Stellenbosch, South Africa \\ Contact address: Willem A Odendaal, Health Systems Research Unit, Medical Research Council of South Africa, Franci van Zyl road, \\ Tygerberg, Western Cape, 7505, South Africa. willem.odendaal@mrc.ac.za.
}

Editorial group: Cochrane Effective Practice and Organisation of Care Group.

Publication status and date: New, published in Issue 11, 2015.

Citation: Odendaal WA, Goudge J, Griffiths F, Tomlinson M, Leon N, Daniels K. Healthcare workers' perceptions and experience on using mHealth technologies to deliver primary healthcare services: qualitative evidence synthesis. Cochrane Database of Systematic Reviews 2015, Issue 11. Art. No.: CD011942. DOI: 10.1002/14651858.CD011942.

Copyright (C) 2015 The Cochrane Collaboration. Published by John Wiley \& Sons, Ltd.

\begin{abstract}
A B S T R A C T
This is the protocol for a review and there is no abstract. The objectives are as follows:

The review has the following two objectives.

- To identify, appraise and synthesise qualitative research evidence on healthcare workers' perceptions and experiences regarding their use of mHealth technologies to provide and support the delivery of primary healthcare services.

- To identify hypotheses, for subsequent consideration and assessment in effectiveness reviews, about why some technologies are more effective than others.
\end{abstract}

\section{B A C K G R O U N D}

Mobile health (mHealth) refers to medical and public healthcare practices supported by mobile devices, such as mobile and smart phones, patient-monitoring devices, personal digital assistants, and tablets (Kay 2011). It also refers to these technologies' capabilities to create, store, retrieve, and transmit information between users (Akter 2010). mHealth relies mainly on the mobile phone's utility of voice, short messaging services (SMS) and multimedia message services (MMS), but also includes more complex applications, such as global positioning systems, bluetooth tech- nology, and third and fourth generation mobile telecommunications (3G and 4G systems) (Kay 2011).

These technologies leverage the reach and speed of mobile networks and mobile computing power to improve the reach of healthcare delivery (Leon 2012; West 2014), including the capturing, processing, and exchange of information (Gagnon 2009), and are transforming the health sector (Qiang 2011; Tomlinson 2013). Its uptake is reflected in the 17 effectiveness reviews published in The Cochrane Library (Appendix 1). These reviews vary in the type and purpose of the technology, from the use of email for 
clinical communication between healthcare professionals (Pappas 2012), to the use of mobile phones for healthcare appointment reminders (Gurol-Urganci 2013). The evidence on the effectiveness of mHealth cited in the reviews, ranges from having no or only a small effect (Braun 2013; Gurol-Urganci 2013), to evidence that it significantly improved targeted health behaviours (Vervloet 2012). The barriers and enablers listed under 'Why is it important to do this review' below, are among the issues that could explain this mixed response to mHealth.

The most common areas to which mHealth is applied are information management, patients' self-management of health and illness, clinical decision support, communication, and service delivery (Blaya 2010; Braun 2013; Catalani 2013; Hall 2014). The proposed review will focus on studies where mHealth has been used to improve healthcare practices and the organisation of care in primary healthcare settings. More specifically, we propose to include studies of healthcare workers' perceptions and experiences of mHealth technologies used for: (i) clinical decision support; (ii) information management used towards organising or delivering services, or both; (iii) communication between healthcare workers as well as between healthcare workers and patients; and (iv) delivery of services. The study will not assess the use of mHealth for patients' self-management of health and illnesses, as the health workers themselves would not be using this technology and therefore their perceptions would not be of direct relevance to this study.

\section{Description of the phenomenon of interest}

For this review, the phenomenon of interest is healthcare workers' perceptions and experiences of their use of mHealth technologies to provide and support primary healthcare services. Healthcare workers will include everyone involved in providing primary healthcare services, including professionals, paraprofessionals, lay health workers, and others, such as managerial and supervisory staff. The following are examples of such programmes.

- Rural healthcare workers have sent pictures and videos of their patients' conditions to urban doctors who reviewed them and returned their diagnoses and treatment guidelines to the healthcare workers. A mHealth application facilitated this communication (West 2014). The perceptions and experiences of both the worker who sent the material and the doctor who replied, are of interest to this review.

- A free open-source mHealth programme assisted 75 lay health workers to manage HIV and TB patients. The lay health workers sent text messages to the programme. The programme recognised key words and sent an automated response which helped the lay health workers (i) to identify patients who needed to be referred; (ii) with drug dosing information; and (iii) with emergency care support (Betjeman 2013). The lay health workers' perceptions and experiences of using these automated responses are of interest to this review.
- A mobile phone application enabled lay health workers to record and track adverse events experienced by MDR-TB multidrug resistant tuberculosis patients and also facilitated real-time lay health worker-doctor communication (Chaiyachati 2013). The perceptions and experiences of both the lay health worker and the doctor about their use of the application are of interest to this review.

- In Project ECHO, specialists offer training and support to primary healthcare providers working in resource-constrained settings through telemedicine, including internet-based assessment tools, online presentations and email communications (Arora 2010). The perceptions and experiences of both the trainer (specialist) and trainee (primary healthcare provider) fall within the scope of this review.

mHealth technologies thus refer to mobile devices that are used to create, store, retrieve and transmit data in real time between users. In addition to the above examples, these technologies also include the following (see Appendix 2 for more details).

- Email and web messaging, to communicate the results of diagnostic medical investigations to patients (Meyer 2012) or clinical communication between healthcare professionals (Pappas 2012); and

- More complex applications, such as global positioning systems (GPS), bluetooth technology, and third and fourth generation mobile telecommunications (3G and $4 \mathrm{G}$ systems) (Kay 2011).

\section{Why it is important to do this review}

The Cochrane and non-Cochrane effectiveness reviews (see Appendix A) not only attest to the ubiquitous and diverse nature of mHealth, but also show mixed results when these types of interventions are used. The evidence of the effectiveness, as provided in the mentioned effectiveness reviews, of any intervention (in this case mHealth) should be supplemented with describing and understanding the barriers and facilitators to the successful implementation of the intervention (Glenton 2013).

This resonates with the conclusions in some of the effectiveness reviews. These suggest that “... patients' and healthcare providers' evaluation and perceptions of the safety of the interventions, potential harms, and adverse effects ... should be assessed” (Gurol-Urganci 2013, p. 2), and “... barriers to trial development and implementation should also be tackled [in future studies]." (Atherton, p. 2). Barriers to the successful implementation of mHealth programmes may include issues tied to the privacy and confidentiality of participant information (Labrique 2013); infrastructural limitations such as reliable network coverage (Aranda-Jan 2014); and poor integration into existing health systems (Aranda-Jan 2014). In contrast, perceptions that the technology empowers the user and improves communication, may 
serve as facilitators to successful implementation of mHealth programmes (Hoffman 2010).

Though there is a growing body of qualitative studies on $\mathrm{mHealth}$, there is still a need for "... social scientific studies explicating processes of technology adoption ...” (Chib 2015, p. 30). Identifying, appraising and synthesising the qualitative evidence of healthcare workers' perceptions and experiences of $\mathrm{mHeal}$ th programmes will complement the reviews of mHealth effectiveness and help improve our understanding of the barriers to and facilitators of its successful implementation (Chaiyachati 2013; Chang 2013; Grimsbø 2012; Medhanyie 2015), as well as helping us to understand the outcomes, implementation and feasibility of these programmes.

\section{O B JECT IVES}

The review has the following two objectives.

- To identify, appraise and synthesise qualitative research evidence on healthcare workers' perceptions and experiences regarding their use of mHealth technologies to provide and support the delivery of primary healthcare services.

- To identify hypotheses, for subsequent consideration and assessment in effectiveness reviews, about why some technologies are more effective than others.

\section{METHODS}

\section{Criteria for considering studies for this review}

\section{Types of studies}

We will include primary studies that use qualitative methods for data collection (for example interviews, focus group discussions, document analysis and observations), and that use qualitative methods for data analysis (for instance, thematic analysis and grounded theory). We will exclude primary studies that collect data using qualitative methods but do not perform a qualitative analysis (e.g. open-ended survey questions where the responses are analysed using descriptive statistics). Mixed methods studies will be included when it is possible to extract data that resulted from the qualitative methods. We will include studies regardless of whether they have or have not been carried out alongside studies of effectiveness of mHealth.

\section{Types of participants}

Participants

We will include studies that focus on the perceptions and experiences of:

- All cadres of healthcare workers (i.e. professionals, paraprofessionals and lay health workers) who are involved in providing primary healthcare services to patients.

- Any other individuals or groups involved in delivering and managing mHealth programmes which aim to provide or support primary healthcare services to patients. These individuals or groups could include administrative staff, information technology staff, managerial and supervisory staff, and may or may not be based in a primary healthcare facility or in the community but must be involved in supporting the delivery of primary healthcare services or the mHealth programmes or both. We will exclude participants identified as technical staff who develop and maintain the mHealth architecture used, for example those involved in writing the software programmes or providing technical support to the end users.

Given the review's focus, i.e. healthcare workers' use of mHealth to deliver primary healthcare services, we will exclude the perceptions and experience of patients in this review.

$\underline{\text { Settings }}$

We will include studies of mHealth programmes that are part of primary healthcare services. For the purposes of this review, we define 'primary healthcare services' as one or any combination of the following:

- the first contact point of healthcare (Awofeso 2004);

- all rehabilitative, therapeutic, preventive and promotive healthcare (Global Health Watch 2011);

- being delivered at an individual or community level or both (Muldoon 2006);

- bringing healthcare services to where people work and live, which in particular applies to low-income settings (Muldoon 2006).

These services can be implemented in public or private primary healthcare facilities, in the community, or the homes of patients. We will include studies conducted in any country.

\section{Phenomena of interest}

The phenomena of interest are healthcare workers' perceptions and experiences regarding their use of mHealth technologies to provide and support primary healthcare services. These technologies refer to mobile devices, such as mobile and smart phones, patient monitoring devices, personal digital assistants, and digital tablets that are used for: (i) clinical decision support; (ii) information management used towards organising or delivering services, or both; (iii) communication between healthcare workers as well as between healthcare workers and patients; and (iv) delivery of primary health care services. In particular $\mathrm{mHealth}$ refers to these 
technologies' capabilities to create, store, retrieve, and transmit information between users.

We will exclude health workers' perceptions of patients' use of mHealth technologies for self-management of health and illnesses as these are not based on their own experience of using the technologies.

\section{Search methods for the identification of studies}

\section{Electronic searches}

We will search PDQ-Evidence http://www.pdq-evidence.org for related reviews in order to identify studies for inclusion.

We will search the following databases for primary studies:

- MEDLINE, Ovid

- CINAHL, EbscoHost

- Global Health, Ovid

- Science Citation Index and Social Sciences Citation Index, ISI Web of Science

(See Appendix 3 for the MEDLINE search strategy.)

As mHealth is a rapidly developing topic, it is likely that many studies are presented at conferences but not yet as journal articles. We will search ISI Index of Conference Proceedings and EMBASE for such conference papers that might not be found elsewhere. Using guidelines developed by the Cochrane Qualitative Research Methods Group for searching for qualitative evidence (Booth 2011), we will develop search strategies for each database. There will be no language limit on the searches. There will be no date or geographic restrictions for the search.

\section{Searching other resources}

We will search the reference lists of all the included studies and key references (i.e. relevant systematic reviews). We will conduct a cited reference search for all included studies in ISI Web of Science and Google Scholar. In addition, we will also search the following grey literature sources.

- Eldis: http://www.eldis.org

- The Grey Literature Report: http://www.greylit.org

- mHealth Database: http://

www.africanstrategies4health.org/mhealth-database.html

- mHealth Evidence: https://www.mhealthevidence.org

- mHealth Knowledge: http://mhealthknowledge.org

- mPowering: http://mpoweringhealth.org

- OpenGrey: http://www.opengrey.eu

\section{Data collection and analysis}

\section{Selection of studies}

The process will comprise the following steps.

Step 1: Assess abstracts and titles according to the inclusion criteria We will collate all titles and abstracts identified through the search strategy into one database. After removing duplicate records and translating non-English records into English, two review authors will assess each record for its potential inclusion eligibility (see Appendix 4 for the proposed development of the screening tool). If necessary, additional staff will be recruited and trained to assist with the abstract and title screening process.

Step 2: Assess full text according to the inclusion criteria

We will exclude records that are not relevant to the topic of this review. Thereafter, we will retrieve the full text of all of the abstracts and titles that have been assessed as potentially eligible. If time and resources allow, we will seek the assistance of colleagues with the relevant language skills to assess non-English texts using the predefined criteria. Where appropriate to further assessment, we will translate these texts into English. Using the same peer review process of two review authors, with the help of additional staff if necessary, we will independently assess the full texts based on the review's inclusion criteria. We will resolve disagreements between authors through discussion, and if they cannot reach consensus, we will refer to the rest of the team for a decision. When appropriate, we will contact study authors for more information to help decide on the inclusion of the study.

Step 3: Potential sampling from the included studies

As qualitative evidence synthesis aims for variation in concepts rather than an exhaustive sample, and because large numbers of studies can impair the quality of the analysis, we will select a sample of studies if more than 40 studies are eligible for inclusion. To allow for the broadest possible variation within the included studies, we will use maximum variation purposive sampling to select from the eligible studies. Key areas of variation that we may consider will include the study methods, the cadre of healthcare worker, the technology used and the purpose of its use, and the geographical setting. Once these variables have been determined, we will create a sampling frame and will map all eligible studies onto the frame. We will then review the studies in each frame, including their number and level of detail, and reach a decision regarding how many studies in each cell we will include in the review.

\section{Data extraction and management}

We will be guided by the Best-fit framework synthesis developed by Carroll and colleagues (Carroll 2013). The process will comprise the following steps.

Step 1: A theoretical model as framework to assess the perceptions and experiences of those who use mHealth

Through consensus, we will identify or develop a theoretical model to capture the salient aspects of the perceptions and experiences of healthcare workers and others, on their use of mHealth technology to deliver primary healthcare services. Current theoretical 
models describe and evaluate the process of adoption and use of technological innovations. For example, the Diffusion of Innovation model (Woodward 2014) emphasises the importance of the socio-cultural context in which technology is used, such as social networks and cultural values, to understand the perceptions and experiences of those who use the technology. Likewise, the Health IT Usability Evaluation model (Brown 2013) offers nine concepts, including the flexibility of the technology to be used for more than one task, that shape users' experiences and perceptions of the technology. Other popular models are the Task-Technology Fit (Smith 2010), Technology Acceptance (Lee 2003), and ICT for Healthcare Development (Chib 2008) models.

Step 2: Development of the data extraction form

The first part of this form will comprise drafting and extracting the study characteristics (see'Appraisal of study quality' below for a list of these characteristics). Each salient concept of the model (Step 1) will be labelled as a theme, each with a detailed definition. Anticipated examples of themes could include: readiness for change, easiness of use; infrastructural barriers; and programme governance. The review authors will independently pilot the model on two included studies and reach consensus on the final version of the model.

Step 3: Coding of the full texts

Each reviewer will code the full texts (manually in Word, using Microsoft Office Professional Plus 2013), using the themes developed in Step 2. Though it is commonly found that the author-findings presented in the 'Results' and 'Discussion' sections contain the data to be coded, we will read all sections for possible coding. Participant quotations will not be treated as data by themselves but will serve as illustrations of authors' descriptions of a phenomenon. Step 4: Revising the theoretical model

Each reviewer will also record data that fall outside the theoretical model (Step 1), which they will share with each other. They will independently assess and label the data, and define the theme. Thereafter they will reach consensus on adding these additional themes to the theoretical model. If needed, the review authors will re-code all data based on such revised theoretical models.

\section{Assessment of risk of bias in included studies}

\section{Appraisal of study quality}

The core quality criterion that a study must meet to be eligible for inclusion, is that it must have used qualitative data collection and analysis methods. We will develop an appraisal form, based on (i) the Critical Appraisal Skills Programme (CASP) (Atkins 2008), and (ii) the methods proposed by Carroll 2012 to assess the quality of the included studies. The form will include, but may not be limited to, the following criteria.

- Adequately described setting and context.

- A well described sampling strategy that is appropriate.
- A well described data collection strategy that is appropriate.

- An adequately described data analysis method that is appropriate.

- Sufficient evidence to support the claims made/findings.

- Adequate evidence of researcher reflexivity.

- Demonstrated sensitivity to ethical concerns.

- Any other concerns raised by the authors.

We will assess each criterion in the adapted CASP tool and then grade each study as having major, moderate or minor methodological limitations.

In finalising the appraisal form, we will pilot it with five included studies. As qualitative studies often do not describe the data analysis in detail, we will use this piloting to decide how to grade short descriptions. Each study will be reviewed by two authors, who will discuss any disagreements between themselves. Unresolved disagreements will be referred to the rest of the author team to be resolved. This peer process of appraisal will apply throughout the review. The methodological appraisal will be used to assess the relative contribution of each primary study in developing the theoretical framework described under 'Data extraction and management' and 'Data synthesis'. The appraisal form will not be used to exclude studies from this review.

\section{Appraisal of the confidence in the review findings}

We will use CERQual (Confidence in the Evidence from Reviews of Qualitative research) to assess the confidence that may be placed in review findings (Lewin 2015). This approach has been developed by the GRADE Working Group 2004. While it is still under development, a standard has been set by previous reviews (Bohren 2015; Colvin 2013; Glenton 2013; Munabi-Babigumira 2015) for its use in assessing the confidence that may be placed in review findings from qualitative evidence syntheses.

This approach uses the following four concepts on which to assess confidence.

- Methodological limitations of included studies: the extent to which there are problems in the design or conduct of the primary studies that contributed evidence to a review finding.

- Relevance of the included studies to the review question: the extent to which the body of evidence from the primary studies supporting a review finding is applicable to the context (perspective or population, phenomenon of interest, setting) specified in the review question.

- Coherence of the review finding: the extent to which the review finding is well grounded in data from the contributing primary studies and provides a convincing explanation for the patterns found in these data.

- Adequacy of the data contributing to a review finding: an overall determination of the degree of richness and quantity of data supporting a review finding.

The above assessments will result in an overall assessment of our confidence in each individual review finding as either high, mod- 
erate, low or very low. We will conclude the appraisal of confidence in each review finding by drafting a table that will summarise the key findings, level of confidence in each, and an explanation for our assessment of each finding.

\section{Data synthesis}

The aim of the data synthesis is to develop a theoretical model we believe to represent a trustworthy, coherent and detailed understanding of the perceptions and experiences of those who deliver and support primary healthcare services. This model will include all initial and newly identified themes ('Data extraction and management', Step 1). As described by Carroll 2013, themes may be grouped together into higher level concepts. We will revisit the evidence used to develop our theoretical model, to graphically display the relationships between the concepts in the model.

This will allow us to hypothesise on whether it is the technology and what it is used for, rather than the type of healthcare worker, or vice versa, or both technology and type of healthcare worker, that shape the perceptions and experiences of healthcare workers. Possible subgroup analyses to test these hypotheses are listed below.

- Are views and perceptions different across different health care provider groups, for example lay health workers' versus health professionals' use of text messaging to communicate with patients?

- Are views and perceptions different across different mHealth technologies,for example the use of email versus text messaging to communicate with patients?

- Are views and perceptions different across different settings (i.e. richer versus poorer, or facility versus community-based), for example the findings of primary studies conducted in low- and middle-income countries versus primary studies conducted in high-income countries?

- Are views and perceptions different when mHealth is used for different health issues, for example the use of text messaging to encourage adherence versus the use of text messaging to communicate test results?

\section{Supplementing the Cochrane effectiveness reviews with} synthesised qualitative findings

Our review findings can be used to contextualise and explain the results of Cochrane mHealth effectiveness reviews and may help to recommend subgroup analysis in future mHealth effectiveness reviews. At present there is no agreed approach on how best to synthesise our results with that of effectiveness reviews. We will explore the appropriateness of using our theoretical model to link our findings on how and why mHealth programmes work to the outcomes described in the Cochrane effectiveness reviews.

\section{Researchers' reflexivity}

Within qualitative research, researchers are expected to reflect on their own background and position, and how it will affect the design, analysis and reporting of their research. We will discuss and describe these issues in a 'Reflexivity' section when publishing our review results.

\section{ACKNOWLEDGEMENTS}

This review is funded by the Alliance for Health Policy and Systems Research, and hosted by the Health Systems Research Unit of the South African Medical Research Council.

Claire Glenton and Simon Lewin of the Oslo satellite of the Cochrane Effective Practice and Organisation of Care Group provided guidance in developing the protocol.

The search strategy was developed by Marit Johansen at the Oslo satellite of the Cochrane Effective Practice and Organisation of Care Group.

Valuable input to improve the protocol was received from the peer referees, Andrew Booth, Meghan Bohren, and Brian McKinstry.

\section{R E F E R E N C S}

\section{Additional references}

\section{Akter 2010}

Akter S, Ray P. mHealth - an ultimate platform to serve the unserved. Yearbook of Medical Informatics 2010;2010: 94-100.

Aranda-Jan 2014

Aranda-Jan CB, Mohutsiwa-Dibe N, Loukanova S.

Systematic review on what works, what does not work and why of implementation of mobile health (mHealth) projects in Africa. BMC Public Health 2014;14:188.
Arora 2010

Arora S, Kalishman S, Thornton K, Dion D, Murata G, Deming P, et al. Expanding access to hepatitis C virus treatment - Extension for Community Healthcare Outcomes (ECHO) project: disruptive innovation in specialty care. Hepatology 2010;52:1124-33.

Atherton

Atherton H, Sawmynaden P, Sheikh A, Majeed A, Car J. Email for clinical communication between patients/ caregivers and healthcare professionals. Cochrane Database of Systematic Reviews 2012, Issue 11. [DOI: 10.1002/ 14651858.CD007978] 


\section{Atkins 2008}

Atkins S, Lewin S, Smith H, Engel M, Fretheim A, Volmink $\mathrm{J}$. Conducting a meta-ethnography of qualitative literature: lessons learnt. BMC Medical Research Methodology 2008;8: 21.

Awofeso 2004

Awofeso N. What is the difference between 'primary care' and 'primary healthcare'?. Quality in Primary Care 2004; 12:93-4.

\section{Betjeman 2013}

Betjeman TJ, Soghoian SE, Foran MP. mHealth in SubSaharan Africa. International Journal of Telemedicine and Applications 2013;2013:6.

Blaya 2010

Blaya JA, Fraser HSF, Holt B. E-health technologies show promise in developing countries. Health Affairs 2010;29: 244-51.

\section{Bohren 2015}

Bohren MA, Vogel JP, Hunter EC, Lutsiv O, Makh SK, Souza JP, et al. The mistreatment of women during childbirth in health facilities globally: a mixed-methods systematic review. PLoS Medicine 2015;12:e1001847.

\section{Booth 2011}

Booth A. Chapter 3: searching for studies. In: Noyes J, Booth A, Hannes K, Harden A, Harris J, Lewin S, Lockwood C editor(s). Supplementary guidance for inclusion of qualitative research in Cochrane systematic reviews of interventions. Cochrane Collaboration Qualitative Methods Group, 2011.

\section{Braun 2013}

Braun R, Catalani C, Wimbush J, Israelski D. Community health workers and mobile technology: a systematic review of the literature. PLoS ONE 2013;8(6):e65772.

\section{Brown 2013}

Brown W, Yen P, Rojas M, Schnall R. Assessment of the Health IT Usability Evaluation Model (Health-ITUEM) for evaluating mobile health (mHealth) technology. Journal of Biomedical Informatics 2013;46:1080-7.

\section{Carroll 2012}

Carroll C, Booth A, Lloyd-Jones M. Should we exclude inadequately reported studies from qualitative systematic reviews? An evaluation of sensitivity analyses in two case study reviews. Qualitative Health Research 2012;22 1425-34.

\section{Carroll 2013}

Carroll C, Booth A, Leaviss J, Rick J. "Best fit" framework synthesis: refining the method. BMC Medical Research Methodology 2013;13:37.

Catalani 2013

Catalani C, Philbrick W, Fraser H, Mechael P, Israelski DM. MHealth for HIV treatment \& prevention: a systematic review of the literature. The Open AIDS Journal 2013;7:17.

Chaiyachati 2013

Chaiyachati KH, Loveday M, Lorenz S, Lesh N, Larkan

L, Cinti S, et al. A pilot study of an mHealth application for healthcare workers: poor uptake despite high reported acceptability at a rural South African community-based MDR-TB treatment program. PLoS ONE 2013;8(5): e64662.

Chang 2013

Chang LW, Njie-Carr V, Kalenge S, Kelly JF, Bollinger RC, Alamo-Talisuna S. Perceptions and acceptability of mHealth interventions for improving patient care at a communitybased HIV/AIDS clinic in Uganda: a mixed methods study. AIDS Care 2013;25:874-80.

Chib 2008

Chib A, Lwin MO, Ang J, Lin H, Santoso F. Midwives and mobiles: using ICTs to improve healthcare in Aceh Besar, Indonesia 1. Asian Journal of Communication 2008; 18:348-64

Chib 2015

Chib A, Van Velthoven MH, Car J. mHealth adoption in low-resource environments: a review of the use of mobile healthcare in developing countries. Journal of Health Communication 2015;20:4-34.

Colvin 2013

Colvin CJ, De Heer J, Winterton L, Mellenkamp M, Glenton C, Noyes J, et al. A systematic review of qualitative evidence on barriers and facilitators to the implementation of task-shifting in midwifery services. Midwifery 2013;29: 1211-21.

Cooke 2012

Cooke A, Smith D, Booth A. Beyond PICO The SPIDER tool for qualitative evidence synthesis. Qualitative Health Research 2012;22:1435-43.

Gagnon 2009

Gagnon M-P, Légaré F, Labrecque M, Frémont P, Pluye P, Gagnon J, et al. Interventions for promoting information and communication technologies adoption in healthcare professionals. Cochrane Database of Systematic Reviews 2009, Issue 1. [DOI: 10.1002/14651858.CD006093.pub2]

Glenton 2013

Glenton C, Colvin CJ, Carlsen B, Swartz A, Lewin S, Noyes $\mathrm{J}$, et al. Barriers and facilitators to the implementation of lay health worker programmes to improve access to maternal and child health: qualitative evidence synthesis. Cochrane Database of Systematic Reviews 2013, Issue 10. [DOI: 10.1002/14651858.CD010414.pub2]

Global Health Watch 2011

Global Health Watch. Primary health care: a review and critical appraisal of its revitalization. http:// www.ghwatch.org/sites/www.ghwatch.org/files/B1_0.pdf 2011; Vol. 3

\section{GRADE Working Group 2004}

GRADE Working Group. Grading quality of evidence and strength of recommendations. BMJ 2004;328:1490-4.

Grimsbø 2012

Grimsbø GH, Engelsrud GH, Ruland CM, Finset A. Cancer patients' experiences of using an Interactive Health 
Communication Application (IHCA). International Journal of Qualitative Studies on Health and Well-being 2012;7:1-14.

\section{Gurol-Urganci 2013}

Gurol-Urganci I, De Jongh T, Vodopivec-Jamsek V, Atun R, Car J. Mobile phone messaging reminders for attendance at healthcare appointments. Cochrane Database of Systematic Reviews 2013, Issue 12. [DOI: 10.1002/ 14651858.CD007458.pub3]

\section{Hall 2014}

Hall CS, Fottrell E, Wilkinson S, Byass P. Assessing the impact of mHealth interventions in low- and middleincome countries - what has been shown to work?. Global Health Action 2014;7:1-12.

\section{Hoffman 2010}

Hoffman JA, Cunningham JR, Suleh AJ, Sundsmo A, Dekker D, Vago F, et al. Mobile direct observation treatment for tuberculosis patients: a technical feasibility pilot using mobile phones in Nairobi, Kenya. American Journal of Preventive Medicine 2010;39:78-80.

Kay 2011

Kay M, Santos J, Takane M. mHealth: new horizons for health through mobile technologies. World Health Organization 2011:66-71.

\section{Labrique 2013}

Labrique AB, Kirk GD, Westergaard RyP, Merritt MW. Ethical Issues in mHealth research involving persons living with HIV/AIDS and substance abuse. AIDS Research and Treatment 2013;2013:1-6.

\section{Lee 2003}

Lee Y, Kozar KA, Larsen KRT. The technology acceptance model: past, present, and future. Communications of the Association for Information Systems 2003;12:50.

\section{Leon 2012}

Leon N, Schneider H, Daviaud E. Applying a framework for assessing the health system challenges to scaling up mHealth in South Africa. BMC Medical Informatics and Decision Making 2012;12:123.

\section{Lewin 2015}

Lewin S, Glenton C, Munthe-Kaas H, Carlsen B, Colvin CJ, Gülmezoglu M, et al. Using qualitative evidence in decision making for health and social interventions: an approach to assess confidence in findings from qualitative evidence syntheses (GRADE-CERQual). PLoS Medicine 2015;12:e1001895.

\section{Medhanyie 2015}

Medhanyie AA, Little A, Yebyo H, Spigt M, Tadesse K, Blanco R, et al. Health workers' experiences, barriers, preferences and motivating factors in using mHealth forms in Ethiopia. Human Resources for Health 2015;13:2.

\section{Methley 2014}

Methley AM, Campbell S, Chew-Graham C, McNally R, Cheraghi-Sohi S. PICO, PICOS and SPIDER: a comparison study of specificity and sensitivity in three search tools for qualitative systematic reviews. BMC Health Services Research 2014;14:579.

\section{Meyer 2012}

Meyer B, Atherton H, Sawmynaden P, Car J. Email for communicating results of diagnostic medical investigations to patients. Cochrane Database of Systematic Reviews 2012, Issue 8. [DOI: 10.1002/14651858.CD007980.pub2]

\section{Muldoon 2006}

Muldoon LK, Hogg WE, Levitt M. Primary care (PC) and primary health care (PHC): what is the difference? - Canadian Journal of Public Health/Revue Canadienne de Santé Publique 2006;97(5):409-11.

\section{Munabi-Babigumira 2015}

Munabi-Babigumira S, Glenton C, Lewin S, Fretheim A, Nabudere H. Factors that influence the provision of intrapartum and postnatal care by skilled birth attendants in low- and middle-income countries: a qualitative evidence synthesis. Cochrane Database of Systematic Reviews 2015, Issue 2. [DOI: 10.1002/14651858.CD011558]

\section{Pappas 2012}

Pappas Y, Atherton H, Sawmynaden P, Car J. Email for clinical communication between healthcare professionals. Cochrane Database of Systematic Reviews 2012, Issue 9. [DOI: 10.1002/14651858.CD007979]

\section{Qiang 2011}

Qiang CZ, Yamamichi M, Hausman V, Altman D, Unit IS. Mobile applications for the health sector. Washington: World Bank 2011.

\section{Smith 2010}

Smith CD, Mentzer JT. Forecasting task-technology fit: the influence of individuals, systems and procedures on forecast performance. International Journal of Forecasting 2010;26: $144-61$.

\section{Tomlinson 2013}

Tomlinson M, Rotheram-Borus MJ, Swartz L, Tsai AC. Scaling up mHealth: where is the evidence?. PLoS Medicine 2013;10(2):e1001382.

\section{Vervloet 2012}

Vervloet M, Linn AJ, Van Weert JCM, De Bakker DH, Bouvy ML, Van Dijk L. The effectiveness of interventions using electronic reminders to improve adherence to chronic medication: a systematic review of the literature. Journal of the American Medical Informatics Association 2012;19: 696-704.

\section{West 2014}

West KL. mHealth: a comprehensive and contemporary look at emerging technologies in mobile health. http:/ /trace.tennessee.edu/cgi/viewcontent. cgi?article $=2711 \&$ context=utk_chanhonoproj 2014:1-12.

\section{Woodward 2014}

Woodward A, Fyfe M, Handuleh J, Patel P, Godman B, Leather A, et al. Diffusion of e-health innovations in 'post-conflict' settings: a qualitative study on the personal experiences of health workers. Human Resources for Health 2014;12:22.

* Indicates the major publication for the study 


\section{A P P E N D I C E S}

\section{Appendix I. Systematic reviews on the effectiveness of mHealth technologies}

Cochrane systematic reviews

1. Atherton H, Sawmynaden P, Sheikh A, Majeed A, Car J. Email for clinical communication between patients/caregivers and healthcare professionals. Cochrane Database of Systematic Reviews 2012a;Issue 11. Art. No.: CD007978. DOI: 10.1002/ 14651858.CD007978.pub2.

2. Atherton H, Sawmynaden P, Meyer B, Car J. Email for the coordination of healthcare appointments and attendance reminders. Cochrane Database of Systematic Reviews 2012b;Issue 8. Art. No.: CD007981. DOI: 10.1002/14651858.CD007981.pub2.

3. de Jongh T, Gurol-Urganci I, Vodopivec-Jamsek V, Car J, Atun R. Mobile phone messaging for facilitating self-management of long-term illnesses. Cochrane Database of Systematic Reviews 2012;Issue 12. Art. No.: CD007459. DOI: 10.1002/ 14651858.CD007459.pub2.

4. Gurol-Urganci I, de Jongh T, Vodopivec-Jamsek V, Car J, Atun R. Mobile phone messaging for communicating results of medical investigations. Cochrane Database of Systematic Reviews 2012;Issue 6. Art. No.: CD007456. DOI: 10.1002/14651858.CD007456.pub2. 5. Gurol-Urganci I, de Jongh T, Vodopivec-Jamsek V, Atun R, Car J. Mobile phone messaging reminders for attendance at healthcare appointments. Cochrane Database of Systematic Reviews 2013;Issue 12. Art. No.: CD007458. DOI: 10.1002/14651858.CD007458.pub3. 6. Lavender T, Richens Y, Milan SJ, Smyth RMD, Dowswell T. Telephone support for women during pregnancy and the first six weeks postpartum. Cochrane Database of Systematic Reviews 2013;Issue 7. Art. No.: CD009338. DOI: 10.1002/14651858.CD009338.pub2. 7. Meyer, B., Atherton, H., Sawmynaden, P, Car, J. Email for communicating results of diagnostic medical investigations to patients. Cochrane Database of Systematic Reviews 2008;Issue 8. CD007980. ISSN 1465- 1858.

8. Pappas Y, Atherton H, Sawmynaden P, Car J. Email for clinical communication between healthcare professionals. Cochrane Database of Systematic Reviews 2012;Issue 9. Art. No.: CD007979. DOI: 10.1002/14651858.CD007979.pub2.

9. Sawmynaden P, Atherton H, Majeed A, Car J. Email for the provision of information on disease prevention and health promotion. Cochrane Database of Systematic Reviews 2012;Issue 11. Art. No.: CD007982. DOI: 10.1002/14651858.CD007982.pub2.

10. Vodopivec-Jamsek V, de Jongh T, Gurol-Urganci I, Atun R, Car J. Mobile phone messaging for preventive health care. Cochrane Database of Systematic Reviews 2012;Issue 12. Art. No.: CD007457. DOI: 10.1002/14651858.CD007457.pub2.

Non-Cochrane systematic reviews

1. Braun R, Catalani C, Wimbush J, Israelski D. Community health workers and mobile technology: A systematic review of the literature. PLoS ONE 2013;8(6): e65772. doi:10.1371/journal.pone.0065772.

2. Free C, Phillips G, Galli L, Watson L, Felix L, Edwards P, et al. The effectiveness of mobile-health technology-based health behaviour change or disease management interventions for health care consumers: A systematic review. PloS Med 2013a;10(1).

3. Free C, Phillips G, Galli L, Watson L, Felix L, Edwards P, et al. The effectiveness of mobile-health technology to improve health care service delivery processes: A systematic review. PloS Med 2013b;10(1).

4. Guy R, Hocking J, Wand H, Stott S, Ali H, Kaldor J. How effective are short message service reminders at increasing clinic attendance? A meta-analysis and systematic review. Health Services Research Health Services Research 2012;47(2):614-632.

5. Källander K, Tibenderana JK, Akpogheneta OJ, Strachan DL, Hill Z, ten Asbroek AHA, et al. Mobile health (mHealth) approaches and lessons for increased performance and retention of community health workers in low- and middle-income countries: A review. Journal of Medical Internet Research 2013;15(1):e17.

6. Shaw R, Bosworth H. Short message service (SMS) as an intervention medium for weight loss. A systematic review. Health Informatics Journal 2012;18(4): 235-250.

7. Vervloet M, Linn AJL, van Weert, JCM, de Bakker DH, Bouy ML, van Dijk L. The effectiveness of interventions using electronic reminders to improve adherence to chronic medication: a systematic review of the literature. Journal of the American Medical Informatics Association 2012;19, 696-704.

Systematic reviews in progress

(Source: http://www.crd.york.ac.uk/PROSPERO/index.asp)

1. Borgstein A, Sondaal S, Browne J, Klipstein K, de Lepper A, Amoakoh-Coleman, M. Systematic review on the role of mHealth in maternal and neonatal care in low and middle income countries. PROSPERO 2014;CRD42014010292.

2. Lunny C, Warje O, Memetovic J. Short message service (SMS) interventions for the prevention and treatment of sexually transmitted infections: a systematic review protocol. PROSPERO 2013;CRD42013006503.

3. Horvath H. mHealth interventions for preventing premature birth. PROSPERO 2014;CRD42014013037.

Healthcare workers' perceptions and experience on using mHealth technologies to deliver primary healthcare services: qualitative 
4. Codyre P, Kalolo A. Use of emerging technologies and mHealth a low resource settings: a systematic review. PROSPERO 2014; CRD42014014282.

5. Lagisetty P, Priyadarshini S, Landgraf J. Evaluating the impact culturally tailored interventions for diabetes prevention. PROSPERO 2015;CRD42015016914.

6. Iribarren S. A systematic review of economic evaluations of mHealth solutions at large in low resource settings/low- and middleincome countries. PROSPERO 2014;CRD42014014913.

7. Gao X, Faber T, Boutron I. Mobile health in developing countries: a systematic review and meta-analysis. PROSPERO 2014; CRD42014008989.

\section{Appendix 2. mHealth technologies (Source: Wikipedia)}

Electronic mail (Email)

It is a method of exchanging digital messages from an author to one or more recipients. Modern email operates across the Internet or other computer networks.

Personal digital assistant (PDA)

Also known as a handheld personal computer or personal data assistant, is a mobile device that functions as a personal information manager. Nearly all PDAs have the ability to connect to the Internet. A PDA has an electronic visual display, enabling it to include a web browser. It also has audio capabilities enabling use as a portable media player, and also enabling most of them to be used as mobile phones. Most PDAs can access the Internet, intranets or extranets via Wi-Fi or Wireless Wide Area Networks. Most PDAs employ touchscreen technology.

Portable media player

Also known as MP3 and MP4 players, it is a portable digital consumer electronics device capable of storing and playing digital media such as audio, images, and video files. The data is typically stored on a CD/DVD, flash memory, micro drive, or hard drive.

Text messaging

Also known as texting, is the act of composing and sending brief, electronic messages between two or more mobile phones, or fixed or portable devices over a phone network. The term originally referred to messages sent using the Short Message Service (SMS). It has grown to include messages containing image, video, and sound content Multimedia Message Service (MMS). BlackBerry Messenger $(\mathrm{BBM})$ is a proprietary Instant Messenger application available for BlackBerry and Android mobile phones.

Web application

It is any software that runs in a web browser.

Web browser

Commonly referred to as a browser, it is a software application for retrieving, presenting and traversing information resources on the World Wide Web.

WhatsApp Messenger

It is an instant messaging app for smartphones that operates under a subscription business model. The proprietary, cross-platform app enables users of select feature phones to use the Internet to communicate.

Other technologies

Handheld video-game consoles, e.g. Playstation Portable, Nintendo DS

Handheld computers e.g. tablets, ipad and Smartbooks

\section{Appendix 3. MEDLINE search strategy}

\begin{tabular}{lll}
\hline$\#$ & Searches & Results \\
\hline 1 & Telemedicine/ & 12815 \\
\hline 2 & Cell Phones/ & 5614
\end{tabular}

Healthcare workers' perceptions and experience on using mHealth technologies to deliver primary healthcare services: qualitative 
(Continued)

\begin{tabular}{|c|c|c|}
\hline 3 & Text Messaging/ & 920 \\
\hline 4 & Electronic Mail/ & 2057 \\
\hline 5 & Computers, Handheld/ & 2606 \\
\hline 6 & MP3-Player/ & 146 \\
\hline 7 & Mobile Applications/ & 577 \\
\hline 8 & Medical Informatics Applications/ & 2159 \\
\hline 9 & Health Information Exchange/ & 172 \\
\hline 10 & $\begin{array}{l}\text { (mobile health or mobile care or mhealth* or } m \text { health*).ti,ab, } \\
\mathrm{kw}\end{array}$ & 1491 \\
\hline 11 & $\begin{array}{l}\text { (electronic health or electronic care or ehealth or e health).ti, } \\
\mathrm{ab}, \mathrm{kw}\end{array}$ & 8848 \\
\hline 12 & $\begin{array}{l}\text { (telemedicine or tele medicine or telehealth or tele health or } \\
\text { telecare or tele care or telemonitoring or tele monitoring).ti,ab, } \\
\mathrm{kw}\end{array}$ & 9840 \\
\hline 13 & (mobile device? or mobile electronic device?).ti,ab,kw. & 1116 \\
\hline 14 & (mobile adj (phone* or telephone*)).ti,ab,kw. & 4177 \\
\hline 15 & cell $^{*}$ phone*.ti,ab,kw. & 2130 \\
\hline 16 & $(($ mobile or cellular) adj (technology or technologies)).ti,ab,kw & 695 \\
\hline 17 & $\begin{array}{l}((\text { mobile or phone or telephone) adj (app? or application?)).ti, } \\
\text { ab,kw }\end{array}$ & 793 \\
\hline 18 & (portable electronic adj (app? or application?)).ti,ab,kw. & 2 \\
\hline 19 & (smartphone* or smart phone?).ti,ab,kw. & 2399 \\
\hline 20 & mobile communication.ti,ab,kw. & 331 \\
\hline 21 & mobile telecommunicat*.ti,ab,kw. & 154 \\
\hline 22 & personal digital assistant?.ti,ab,kw. & 942 \\
\hline 23 & patient monitor* device?.ti,ab,kw. & 22 \\
\hline 24 & text messag*.ti,ab,kw. & 1560 \\
\hline
\end{tabular}


(Continued)

\begin{tabular}{lll}
25 & (electronic mail? or email? or e mail?).ti,ab,kw. & 8696 \\
\hline 26 & short messag* service?.ti,ab,kw. & 108 \\
\hline 27 & (sms adj (messag* or service*)).ti,ab,kw. & 37 \\
\hline 28 & ((multi media or multimedia) adj messag* service?).ti,ab,kw. & 7 \\
\hline 29 & (mms adj (messag* or service*)).ti,ab,kw. & 17 \\
\hline 30 & web messaging.ti,ab,kw. & 21 \\
\hline 31 & (whatsapp or whats app).ti,ab,kw. & 138 \\
\hline 32 & (instant messaging or instant messenger).ti,ab,kw. & 635 \\
\hline 33 & ((handheld or hand held) adj computer?).ti,ab,kw. & 37 \\
\hline
\end{tabular}

34 (computer tablet? or pc tablet? or palmtop computer? or palm 671 top computer? or pda computer? or pocket pc? or pda phone or blackberry or palm pilot? or pilot palm?).ti,ab,kw

35 ((handheld or hand held) adj3 console?).ti,ab,kw. 8

36 (mp3player? or mp3 player? or mp4player? or mp4 player?).ti, 98 $\mathrm{ab}, \mathrm{kw}$

37 (ipod or ipods or i pod or i pods).ti,ab,kw.

38 portable media player?.ti,ab,kw.

15

39 pager?.ti,ab,kw.

40 global position? system?.ti,ab,kw.

41 ((3G or $4 \mathrm{G})$ adj system?).ti,ab,kw. 7

42 (bluetooth technolog* or blue tooth technolog*).ti,ab,kw. 25

43 (videoconsult* or video consult*).ti,ab,kw. $^{*} 115$

$\begin{array}{ll}44 \text { or } / 1-43 & 49806\end{array}$

45 (qualitative or themes).mp. 169392

4644 and $45 \quad 2269$

Healthcare workers' perceptions and experience on using mHealth technologies to deliver primary healthcare services: qualitative 


\section{Appendix 4. 'SPIDER abstract/title screening'}

We will use SPIDER (Cooke 2012; Methley 2014) to develop the screening tool, comprising the following elements:

\begin{tabular}{ll}
\hline Sample & $\begin{array}{l}\text { Healthcare workers and other individuals involved in providing primary healthcare services, including: } \\
\cdot \text { professionals } \\
\cdot \text { paraprofessionals } \\
\cdot \text { lay health workers } \\
\cdot \text { programme and health service managers } \\
\cdot \text { staff supervisors } \\
\cdot \cdot \text { information technology staff }\end{array}$ \\
\hline Phenomenon of Interest & $\begin{array}{l}\text { Healthcare workers'other individuals' perceptions and experiences regarding their use of mHealth tech- } \\
\text { nologies to provide and support primary healthcare services }\end{array}$ \\
\hline Design & $\begin{array}{l}\text { Data collection methods will include but may not be limited to: } \\
\cdot \text { interviews, focus group discussions, document analysis and observations } \\
\text { Data analysis methods will include but may not be limited to: } \\
\cdot \text { thematic analysis and grounded theory }\end{array}$ \\
\hline Evaluation & \begin{tabular}{l} 
Experiences and perceptions \\
\hline Research type
\end{tabular} \\
\hline
\end{tabular}

\section{CONTRIBUTIONS OFAUTHORS}

Led by Willem Odendaal, the review was collectively conceptualised by the full author team. Willem Odendaal drafted the first version of this protocol. Jane Goudge, Frances Griffiths, Mark Tomlinson and Karen Daniels reviewed and commented on all drafts of this protocol, and Natalie Leon engaged with later drafts.

\section{DECLARATIONS OF INTEREST}

Willem A Odendaal: none

Jane Goudge: none

Frances Griffiths: none

Mark Tomlinson: None

Natalie Leon: none

Karen Daniels: none 


\section{SOURCES OF SUPPORT}

\section{Internal sources}

- No sources of support supplied

\section{External sources}

- Alliance for Health Policy and Systems Research, Other. 